\title{
FRACTIONNEMENT DES ESTERS MÉTHYLIQUES DU BEURRE PAR CHROMATOGRAPHIE SUR COUCHE MINCE DE SILICAGEL IMPRÉGNÉ DE NITRATE D'ARGENT
}

\section{I. ÉTUdE PRÉliMINAIRE}

par

\section{Simonne KUZDZAL-SAVOIE}

(Avec la collaboration technique de Janine Trehin)

Station Centrale de Recherches Laitières et de Technologie des Produits animaux, Jouy-en-Josas (Yvelines - France)

\section{Introduction}

Le fractionnement sur couche mince de silicagel imprégné de nitrate d'argent des esters méthyliques totaux du beurre a permis d'isoler les esters d'acides gras trans du beurre (Kuzdzal-Savoie et al., 1966 ; Perron et al., 1967).

Mais d'autres groupes d'acides gras sont aussi isolés et ce fractionnement peut être considéré comme une méthode préliminaire générale facilitant l'étude ultérieure de certains acides gras mineurs du beurre.

Au cours du présent travail, nous avons recueilli à partir des esters totaux du beurre diverses fractions que nous avons soumises à un premier examen. Nous avons en outre cherché à améliorer la résolution du fractionnement.

\section{Méthode expérimentale}

Le beurre utilisé dans la présente étude a été fabriqué selon le procédé industriel à Surgères (Charente-Maritime) le 5 mai 1966.

\section{A) Fractionnement.}

Nous avons successivement pratiqué le fractionnement classique à développement vertical sur plaques de $20 \times 20 \mathrm{~cm}$ (essai 1) et le fractionnement à développement horizontal sur plaques de $20 \times 40 \mathrm{~cm}$ (essai 2), pour lequel nous avons utilisé après l'avoir modifié, l'appareillage B. N. de Desaga.

La préparation des plaques est semblable dans les deux cas : nitrate d'argent, silicagel $G$ et eau sont utilisés dans le rapport $1 / 3 / 6$, 
l'épaisseur de la couche est de $1 \mathrm{~mm}$ pour les petites plaques et de $0,5 \mathrm{~mm}$ pour les grandes plaques. Le dépôt est effectué par points ou par bandes et les esters sont utilisés à une concentration de 6 ou 10 p. 100. Le solvant de développement est un mélange benzène/éther de pétrole $(80 / 20)$. Le développement dure 30 minutes (essai 1) ou 2 h - 2 h 30 (essai 2 ).

Les chromatoplaques sont révélées sous U.V. après vaporisation par une solution de 2,7-dichlorofluorescéine à 0,2 p. 100 dans l'éthanol. Les révélations aux vapeurs d'iode ou à l'acide phosphomolybdique (solution à 20 p. 100 dans l'éthanol) ont été parfois utilisées.

\section{B) Étude des fractions.}

Les fractions d'esters sont recueillies au moyen d'un système d'aspiration automatique, dans une cartouche de cellulose. Celle-ci est ensuite placée dans un appareil B.B.S. d'extraction en continu. L'oxyde d'éthyle est utilisé comme solvant d'extraction.

a) Spectrophotométrie infra-rouge.

Les esters sont déposés sur les fenêtres de chlorure de sodium sous forme de films de moins de $0,025 \mathrm{~mm}$ d'épaisseur. L'étude de la zone 10-11 $\mu$ permet de déterminer la présence ou l'absence de liaisons trans.

b) Chromatographie en phase gazeuse.

Les esters recueillis sont directement injectés dans l'appareil de chromatographie en phase gazeuse : Aerograph $\mathrm{Hy} \mathrm{Fi}-600-\mathrm{C}$, à détecteur à ionisation de flamme, équipé d'une colonne de $3 \mathrm{~m}$ de long et de $0,3 \mathrm{~cm}$ de diamètre intérieur, chargée à 10 p. 100 de succinate de diéthylène glycol sur chromosorb W lavé aux acides (80/100 mesh), et chauffée à une température voisine de $150^{\circ} \mathrm{C}$ ou de $170^{\circ} \mathrm{C}$. On a également utilisé le succinate de butane diol (polyester de Craig) dans les mêmes conditions que le succinate de diéthylène glycol.

A diverses reprises des esters connus ont été ajoutés aux fractions étudiées pour faciliter la reconnaissance des pics.

\section{Résultats}

\section{A) Résolution.}

Deux chromatoplaques obtenues, l'une dans les conditions de l'essai 1 et l'autre dans les conditions de l'essai 2, sont présentées respectivement sur les figures 1 et 2 . Sur la chromatoplaque de la figure 2 apparaît également l'image du fractionnement des esters méthyliques d'un échantillon de saindoux effectué simultanément au fractionnement des esters du beurre. 
On observe une amélioration de la résolution de l'essai 1 à l'essai 2. L'espace séparant les monoèmes-trans des monoèmes-cis est, dans l'essai 2, relativement grand ce qui permet un bon isole-

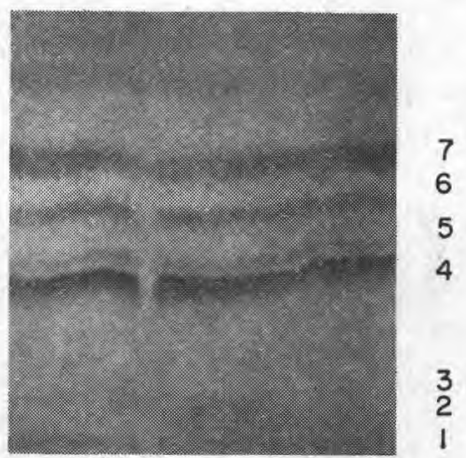

Fig. 1. - Fractionnement des esters méthyliques du beurrè dans les conditions de l'essai 1 . Les fractions recueillies sont numérotées de 1 à 7 .

1 : esters polaires et triènes; 2 : diènes ; $3:$ ?;

4: monoènes-cis ; $5:$ ?; 6 : monoènes-trans; 7 : saturés.

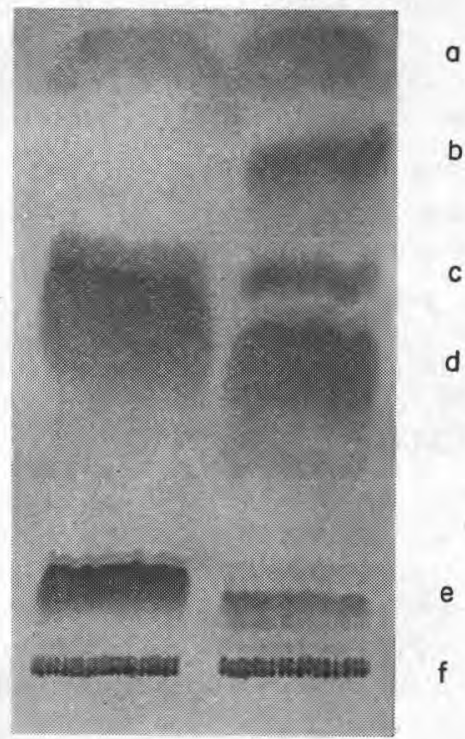

$\mathrm{Sa}$

$\mathrm{Be}$

Fig. 2. - Fractionnement des esters méthyliques du beurre (Be) et du saindoux (Sa) dans les conditions de l'essai 2.

$a$, saturés et ramifiés; b, monoènes trans; c, diènes conjugués (?) ; $\mathrm{d}$, monoènes-cis; e, diènes non conjugués; $f$, triènes et esters polaires. 
ment de la bande intermédiaire. Deux bandes mineures supplémentaires ont été observées lors du fractionnement des esters méthyliques du beurre, l'une située juste au-dessus des cis-monoènes, l'autre juste au-dessous des monoènes trans. Ces bandes mineures n'apparaissent pas sur la figure 2.

Au niveau des diènes on observe, pour le beurre, quatre zones ou bandes d'intensité variable.

La technique de fractionnement proposée facilite done l'étude détaillée des acides gras du beurre puisqu'elle permet d'obtenir d'une manière relativement simple des groupes d'acides gras théoriquement plus homogènes que le mélange de départ.

\section{B) Étude des fractions obtenues dans les conditions de l'essai 1.}

Les résultats pondéraux obtenus au cours de onze opérations successives de fractionnement et de récupération selon l'essai 1 , sont portés dans le tableau 1. A chaque opération, 7 fractions furent recueillies, désignées de F1 à F7, depuis le bas de la plaque jusqu'à l'extrémité supérieure. On constate que la reproductibilité du fractionnement est relativement bonne mais l'analyse n'est que semi-quantitative. Les zones correspondant aux acides polyènes sont d'ailleurs assez difficiles à délimiter, car on observe plusieurs bandes. De plus, les composés restant à l'origine sont incorporés à la fraction Fl (qui comprend également les triènes).

Les différentes fractions recueillies au cours de l'essai 1 (première opération) ont été soumises à un rapide examen.

a) Spectrophotométrie infra-rouge.

Une bande intense a été observée à $10,36 \mu$ pour la fraction $\mathrm{F} 6$, que nous savons être constituée par les esters d'acides gras trans.

La fraction F5 présente également une absorption à $10,36 \mu$ mais celle-ci est complétée par une absorption à $10,18 \mu$; à $10,54 \mu$ on décèle une très légère inflexion de la courbe. Les fractions F1, F2 et F3 provenant de la région, des polyènes montrent une légère absorption vers $10,36 \mu$.

b) Chromatographie en phase gazeuse.

La fraction F7 est constituée par les acides saturés à chaîne droite auxquels s'ajoutent les acides ramifiés facilement reconnaissables par leurs temps de rétention, bien connus dans le cas du beurre. Le rapport acides ramifiés/acides saturés à chaîne droite est, dans la fraction F7, approximativement égal à ce qu'il est dans les esters totaux. Les acides ramifiés se retrouvent done, pratiquement en totalité, avec les acides saturés à chaîne droite. Dans le beurre les acides ramifiés connus sont d'ailleurs tous saturés (Iverson et al., 1965).

Le chromatogramme des acides gras trans du beurre, isolés par chromatographie sur couche mince (fraction F6) est analogue 
au chromatogramme des acides trans isolés par chromatographie sur colonne (Kuzdzal-Savoie et Raymond, 1965) : pic majeur 18: 1, comportant un pic mineur adjacent (dont le temps de rétention est légèrement plus élevé), présence de pies dont les temps de rétention sont analogues à ceux des acides $16: 1$ et $17: 1$.

De même le chromatogramme de la fraction F4 est analogue à celui obtenu antérieurement à partir des esters monoènes-cis, isolés par chromatographie sur colonne (Kuzdzal-Savoie et Raymond, 1965). L'acide monoène à 18 atomes de carbone correspond à environ 90 p. 100 des acides de cette fraction. On observe également les pics correspondant aux acides mineurs monoènes à 14,16 et 17 atomes de carbone. Cependant un pic supplémentaire dont le temps de rétention est voisin de celui de l'acide iso 16:0 apparaît sur le chromatogramme de la fraction, F4.

La fraction F5 est constituée par la zone qui s'étend entre les monoènes-trans et les monoènes-cis. Dans cette zone apparaît une bande nettement individualisée.

Les 11 fractions F5 recueillies au cours des opérations successives de fractionnement dans les conditions de l'essai 1 ont été réunies. L'analyse par chromatographie en phase gazeuse, conduite en utilisant du succinate de butane diol comme phase stationnaire, a permis d'obtenir le chromatogramme reproduit sur la figure 5 . On observe un pic 18: 1 relativement important et 4 pies (numérotés de 1 à 4 ) à temps de rétention élevé.

Dans les conditions de l'analyse chromatographique utilisées ici, les longueurs de chaîne équivalentes correspondant aux pios 1 à 4 sont : 19,55 - 19,75 - 20,1 et 20,4. Dans les mêmes conditions d'analyse les longueurs de chaîne équivalentes des acides linoléique et linolénique sont respectivement de 18,75 et de 19,25.

Le chromatogramme de la fraction F2 (également porté sur la figure 3 ) comporte un double pic majeur qui se place au niveau des diènes à 18 atomes de carbone.

Dans la fraction F1 un pic notable est observé au niveau des triènes à 18 atomes de carbone, mais de nombreux autres pies sont présents, en particulier des pies dont les temps de rétention sont voisins de ceux des acides à 14,16 ou 17 atomes de carbone.

\section{Discussion}

Comme nous l'avons annoncé dans l'introduction le travail présenté constitue une étude préliminaire.

Il nous a permis de fractionner les esters méthyliques totaux du beurre en groupes comportant un nombre restreint d'acides gras de nature relativement homogène, sur lesquels une étude ultérieure peut être engagée.

Ainsi le présent travail nous a permis d'isoler un groupe d'acides 
TABLEAU

FRACTIONNEMENT DES

\begin{tabular}{|c|c|c|c|c|c|c|c|c|c|c|c|}
\hline \multirow{3}{*}{$\begin{array}{c}\text { fractions } \\
7\end{array}$} & \multirow{3}{*}{$\begin{array}{l}\text { Dénomination } \\
\text { Saturés }\end{array}$} & \multirow{2}{*}{\multicolumn{2}{|c|}{$\mathrm{mg}^{1} \%$}} & \multirow{2}{*}{\multicolumn{2}{|c|}{$\mathrm{mg}^{2}$}} & \multicolumn{2}{|c|}{3} & \multicolumn{2}{|c|}{4} & \multicolumn{2}{|c|}{5} \\
\hline & & & & & & mg & $\%$ & $\mathrm{mg}$ & $\%$ & & $\%$ \\
\hline & & 80,2 & 49,3 & 146,1 & 52,5 & 133,3 & 54,2 & 158,0 & 58,8 & 187,2 & 58,2 \\
\hline 6 & Monoènes trans & 12,5 & 7,7 & 19,4 & 7,0 & 18,5 & 7,5 & 22,0 & 8,2 & 23,7 & 7,4 \\
\hline 5 & $?$ & 7,2 & 4,4 & 9,0 & 3,2 & 6,6 & 2,7 & 10,9 & 4,1 & 9,8 & 3,1 \\
\hline 4 & Monoènes Cis & 47,6 & 29,3 & 67,1 & 24,2 & 62,1 & 25,2 & 58,1 & 21,8 & 72,9 & 22,8 \\
\hline $\begin{array}{l}3 \\
2\end{array}$ & Diènes & 9,2 & 5,7 & 19,2 & 6,9 & 14,6 & 5,9 & 8,6 & 3,2 & 14,6 & 4,6 \\
\hline 1 & Polyènes & 5,8 & 3,6 & 17,3 & 6,2 & 11,1 & 4,5 & 10,6 & 3,9 & 12,4 & 3,9 \\
\hline & Total & 162,5 & 100 & 278,1 & 100 & 246,2 & 100 & 268,1 & 100 & 320,6 & 100 \\
\hline
\end{tabular}

gras (situé entre les cis-monoènes et les trans-monoènes) sur lequel nous porterons en premier lieu notre attention.

Les études par chromatographie sur couche mince de silicagel imprégné de nitrate d'argent réalisées à partir d'esters connus (De Vries et Jurriens, 1963; de Jong et Van der Wal, 1964; Pallota et al., 1965 ; Morris, 1966) montrent qu'un certain nombre d'acides peuvent, sous forme de leurs esters méthyliques se placer entre les cis-monoènes et les trans-monoènes. Ces acides peuvent être des isomères de position de l'acide oléique dont la double liaison est plus éloignée du carboxyle que le carbone 9 , des acides à plus longue chaîne ( 20 ou 22 atomes de carbone), dont la double liaison cis est aussi plus éloignée du carboxyle que le carbone 9 . Il peut s'agir également d'acides monoènes trans à 18 atomes de carbone dont la double liaison, serait plus proche du carboxyle que le trans monoène majeur du beurre (trans, 11-octadécénoïque), ou d'acides monoènes trans à plus courte chaîne. Enfin parmi les acides diènes citons les acides à double liaisons non conjuguées trans-trans (ils seraient alors très proches des monoènes-cis) et les acides à doubles liaisons conjuguées cis-trans ou trans-trans 


\begin{tabular}{|c|c|c|c|c|c|c|c|c|c|c|c|c|}
\hline \multicolumn{2}{|c|}{6} & \multicolumn{2}{|c|}{7} & \multicolumn{2}{|c|}{8} & \multicolumn{2}{|c|}{9} & \multicolumn{2}{|c|}{10} & \multicolumn{2}{|c|}{11} & \multirow{2}{*}{ Moyenne } \\
\hline $\mathrm{mg}$ & $\%$ & $\mathrm{mg}$ & $\%$ & $\mathrm{mg}$ & $\%$ & $\mathrm{mg}$ & $\%$ & $\mathrm{mg}$ & $\%$ & $\mathrm{mg}$ & $\%$ & \\
\hline 259,5 & 51,7 & 261,2 & 54,4 & 324,3 & 57,0 & 156,2 & & 215,6 & 55,6 & 374,5 & 54,2 & 54,6 \\
\hline 44,6 & 8,9 & 38,8 & 8,1 & 44,4 & $-7,8$ & 26,0 & & 28,6 & 7,4 & 59,1 & 8,5 & 7,8 \\
\hline 18,9 & 3,8 & 15,5 & 3,2 & 14,2 & 2,5 & 8,9 & & 11,0 & 2,8 & 32,3 & 4,7 & 3,5 \\
\hline 115,4 & 23,1 & 114,0 & 23,9 & 136,6 & 24,0 & $(21,1)$ & & 96,3 & 24,9 & 162,6 & 23,4 & 24,3 \\
\hline 41,9 & 8,4 & 31,0 & 6,4 & 31,7 & 5,5 & 30,4 & & 24,8 & 6,4 & 42,6 & 6,2 & 5,9 \\
\hline 20,6 & 4,1 & 19,4 & 4,0 & 18,3 & 3,2 & 17,2 & & 11,3 & 2,9 & 20,6 & 3,0 & 3,9 \\
\hline 500,9 & 100 & 479,9 & 100 & 569,5 & 100 & 259,8 & & 387,6 & 100 & 691,7 & 100 & 100 \\
\hline
\end{tabular}

(ces derniers seraient alors très proches des monoènes trans, les diènes cis-trans se plaçant un peu au-dessous).

Compte tenu des résultats que nous avons obtenus au cours des analyses spectrophotométriques et chromatographiques et en nous appuyant sur les études de Scott et al. (1959), de Litchfield et al. (1963), de Body et Shorland (1965), nous pensons que la fraction F5 contient une forte proportion d'acides diènes conjugués, ceux-ci se trouvant groupés sur la chromatoplaque, dans la bande centrale de la zone qui s'étend des monoènes trans aux monoènes cis, et apparaissant sur le chromatogramme de la fraction F5 au niveau des pies numérotés de 1 à 4 .

Un argument en faveur de cette hypothèse est donné par l'image du fractionnement des esters méthyliques du saindoux; 2 bandes fines surmontent l'ester de l'acide oléique mais aucune bande n'apparaît dans la zone des diènes conjugués. Or Iverson et al. (1965) ont récemment démontré l'absence de ces composés dans le saindoux.

Les acides diènes conjugués du beurre n'ont pas été antérieurement isolés. L'emplacement des doubles liaisons n'a pas, à notre 

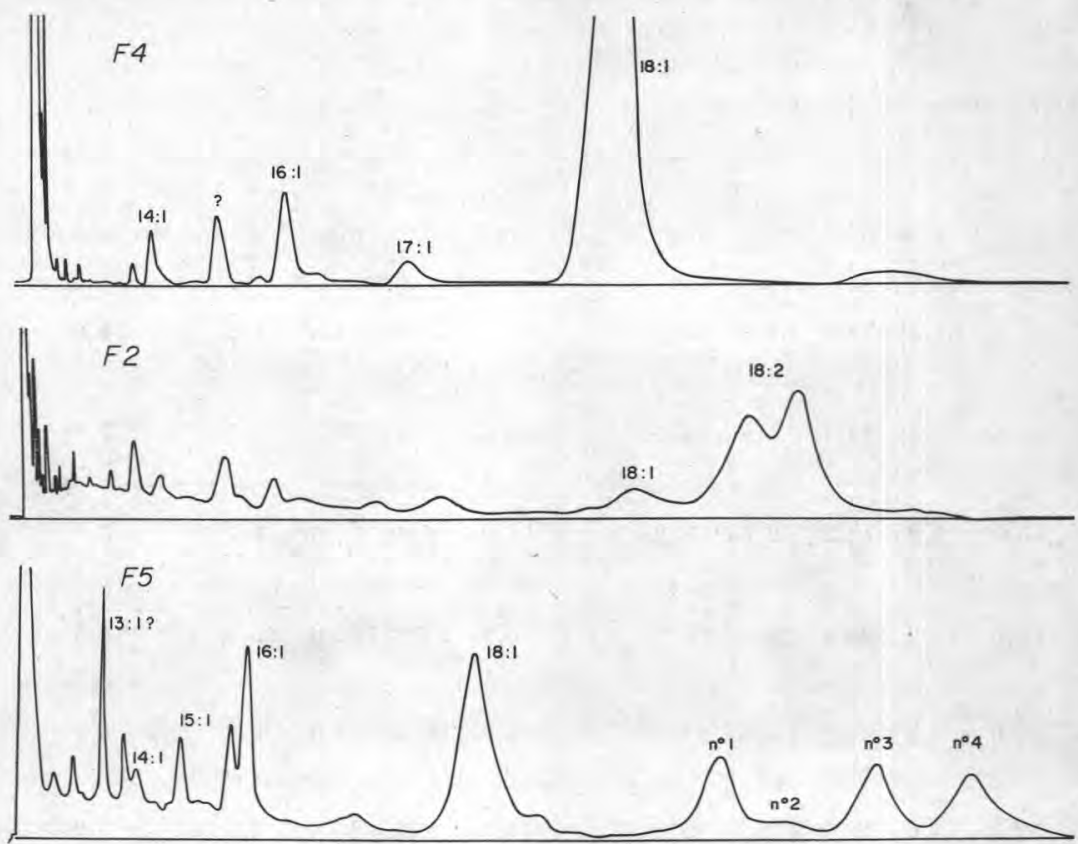

Fig. 3. - Chromatogrammes des fractions F4, F2 (première opération) et F5 (11 opérațions réunies) obtenues dans les conditions de l'essai 1. (Cf. tableau I).

Phases stationnaires : pour F4 et F2, succinate de diéthylène glycol; pour F5, succinate de butane diol.

connaissance été précisé. Par contre Smith et al. ont dès 1954 indiqué que les acides diènes du beurre présentaient la configuration cis-trans et trans-trans, la première configuration étant prépondérante. Ceci a été confirmé par Herb et al., en 1962, à l'aide de la chromatographie en phase gazeuse. Jusqu'à maintenant les acides diènes conjugués cis-cis à 18 atomes de carbone n'ont été trouvés dans aucun produit naturel (Body et Shorland, 1965). La présence de ceux-ci dans le beurre a cependant été suggérée (KuzdzalSavoie et al., 1966).

Il convient donc d'étudier d'une manière approfondie les constituants de la fraction F5. On utilisera pour cela les conditions de meilleure résolution proposées dans l'essai 2, lors du fractionnement des esters méthyliques du beurre sur couche mince de silicagel imprégné de nitrate d'argent, et les méthodes d'identification spectrophotométriques et chromatographiques appropriées.

Les conditions dans lesquelles se déroulent le fractionnement sur couche mince, la récupération des esters et leur analyse, sont très favorables au développement de l'oxydation. Celle-ci peut 
entraîner la formation d'acides gras différents de ceux existants à l'origine dans le beurre. Toute précaution devra être prise dans ce domaine afin d'éviter une interprétation erronée des résultats.

(Reçu pour publication en octobre 1967)

\section{Résumé}

Le fractionnement des esters méthyliques du beurre par chromatographie sur couche mince de silicagel imprégné de nitrate d'argent, permet d'isoler les groupes d'acides gras suivants : saturés (et ramifiés); monoènes trans, diènes conjugués (?), monoènes-cis, diènes non conjugués, triènes et acides polaires.

Dans des conditions de résolution améliorée (chromatographie horizontale sur plaque de $40 \mathrm{~cm}$ de long), le fractionnement peut être utilisé à l'étude des acides gras mineurs présents dans les différents groupes.

L'étude des acides diènes conjugués du beurre constitue un exemple d'application de la méthode proposée.

\section{Sum mary}

By fractionating methyl esters of butter by thin layer chromatography on silver nitrate impregnated silicagel, the following groups of fatty acids have been separated : saturated (and branched); monoene-trans, diene conjugated (?), monoene-cis, diene non conjugated, triene and polar acids.

The resolution conditions being improved (horizontal chromatography on $40 \mathrm{~cm}$ long plates), the fractionating may be applied to the study of minor fatty acids in the different groups.

The method proposed may for instance be used for studying the diene conjugated acids of butter.

\section{RÉFÉRENCES BIBLIOGRAPHIQUES}

Body (D. R.) et SHorland (F. B.), 1965. The geometric isomers of conjugated otadecadienoates from dehydrated methyl ricinoleate. J. Amer. Oil Chem. Soc., 42, 5.

De Vries (B.) et JuRriens (G.), 1963. Trennung von lipiden mittels dünnschicht chromatographie auf mit silbernitrat impragnierten Kieselgel. Fette Seifen Anstrich, 65, 725.

Herb (S. F.), Magidman (P.), Luddy (F. E.) et Riemenschneider (R. W.), 1962. Fatty acids of cows'milk. B. Composition by gas-liquid chromatography aided by other methods of fractionation. J. Amer. Oil. Chem. Soc., $39,142$. 
Iverson (J. L.), Eisner (J.), Firestone (D.), 1965. Detection of trace fatty acids in fats and oils by urea fractionation and gas liquid chromatography. J. Amer. Oil Chem. Soc., 42, 1063.

DE JONG K. et VAN DER WEL (H.), 1964. Identification of some iso-linoleic acids occurring in butter fat. Nature, 202, 553.

KuzdzaL-SAvole (S.) et RAYmond (J.), 1965. Les acides gras trans du beurre. I. Isolement et dosage. Ann. Biol. Anim. Biophys. Bioch., 5, 497.

Kuzdzal-Savoie (S.), Raymond (J.), Kuzdzal (W.) et Petit (J.), 1966. Les acides gras trans du beurre. II, Contribution à l'étude de leur origine. Ann. Biol. Anim. Bioch. Biophys., 8, 351.

Litchfield (C.), Reiser (R.) et Isbeli (A. F.), 1963. The analysis of cistrans fatty acid isomers using gas liquid chromatography. $J$. Amer. Oil Chem. Soc., 40, 302.

Morris (L. J.), 1966. Separations of lipids by silver ion chromatography. J. Lip. Res., 7, 717.

Pallota (U.), Losi (G.) et Zorzut (C.), 1965. Sulla determinazione dell' acido elaidinico negli olu di crucifere. Riv. Ital. Sost. Grasse, 42, 142.

Perron (R.), Blanchard (P.), Chaveron (H.) et Auffret (M.), 1967. Méthode de détermination précise de l'acide élaïdique dans les matières grasses, Rev. Franç. Corps Gras, 14, 241.

Scott (W. E.), Herb (S. F.), Magidman (P.) et Riemenschneider (R. W.), 1959. Unsaturated fatty acids of butter fat. J. Agri. Food Chem., 7, 125.

Smith (L. M.), FreEmann (K.) et JACK (E. L.), 1954. The unsaturated fatty acid of milk fat. III. Geometrical isomerism. J. Dairy Sci., 37, 399. 